\title{
Evaluation Performance of Protocols LEACH, 802.15.4 and CBRP, Using Analysis of QoS in WSNs
}

\author{
Syyed Javad Mohammadi Baygi, Mehran Mokhtari \\ Department of Computer Engineering, Islamic Azad University, Damghan Branch, Damghan, Iran \\ Email: mohammadi.s.j@gmail.com, mehrmokhtari@yahoo.com
}

Received 27 August 2014; revised 26 September 2014; accepted 27 October 2014

Copyright (C) 2014 by authors and Scientific Research Publishing Inc.

This work is licensed under the Creative Commons Attribution International License (CC BY). http://creativecommons.org/licenses/by/4.0/

(c) (i) Open Access

\begin{abstract}
Standards can define the performance and required protocols in different types of nodes in Wireless Sensor Networks (WSNs). In this article we discuss the Quality of Service (QoS) requirements in WSNs and present a survey of some of the QoS multi-parameters metrics in WSNs. Also, it gives the performance of 802.15.4, LEACH, and CBRP protocol considering parameters. The simulation tests were carried out by the simulator NS 2.29; it showed that the performance of 802.15.4 protocol is better than the LEACH. The results have shown that the 802.15.4 standard protocol provides better quality of outperformed service requirements than LEACH protocol. So its performance in WSNs is more effective than LEACH protocol. CBRP protocol simulators NS 2.34, reduce traffics and congestion packet. Finally, the algorithm CBRP offers optimal performance with reduced overhead and delay with respect to the other two protocols. Plus, the strengths and weaknesses of the protocols are specified in relation to the provision of quality of service. It should take effective action to address them.
\end{abstract}

Keywords

802.15.4, LEACH, CBRP, QOS, WSN

\section{Introduction}

Wireless Sensor Networks (WSNs) have been identified as one of the most important technologies of the 21st century, and these networks are a new member of wireless networks' family. They have a series of specific needs, and characteristics [1]. Therefore, WSNs consist of a large number of nodes in a particular area. Each of them is able to collect information such as temperature, pressure, humidity, noise, light, etc., from the areas 
where they are located. They send the collected data to the sink nodes [2]. Quality of Service (QoS) in WSNs is very different from that in traditional networks [3]. QoS (acceptable delay limit, packet loss, etc.) is important depending on the application. Therefore, it is important to pay attention to these standards for the design and selection of clustering method. For example, RFC 2386 considers the QoS as a set of service requirements [4].

They should be considered when delivering a packet from source to the destination. In this scenario, the service quality is defined as:

The internet is guaranteed to provide a set of properties of appropriate services for users, or end-to-end applications based on the delay, jitter, bandwidth and packet loss. There are two approaches to service quality that can be shown by a simple model [5] [6]. These models are shown in Figure 1.

QoS parameters of WSNs may have different types, which depend on network application. Some of the parameters are used to evaluate the quality of the service such as: the whole network covering, the optimal number of active nodes in the network, the accuracy of the observation (measurement accuracy of the sensor), the accuracy of the data transfer, data transfer in the shortest possible time, reliability, network lifetime and energy consumption in the networks. The main purpose of this article is to compare the performance of three protocols (802.15.4, LEACH, and CBRP) in WSNs. Simulation and implementation have been done on the three protocols in NS 2.29 and NS 2.34 simulator under Linux.

A number of scenarios are designed. The results are compared with each other, and are analyzed. The QoS parameters are compared with each other. Then, the performances of the protocol 802.15.4, LEACH and CBRP are evaluated in the network topology. Also, in this stage, strengths and weaknesses of protocols are identified, and we can take the necessary action to resolve them. Then, in Section 2, 802.15.4 protocol is reviewed; in Section 3, LEACH protocol, in Section 4, CBRP protocol, in Section 5, the related work, in Section 6, existing requirements \& challenges for QoS in WSNs, in Section 7, simulation setup and performance metrics analysis, in Section 8, result and discussion, in Section 9, comparison of performance evaluation 802.15.4 and LEACH with CBRP, in Section 10 conclusion, are presented.

\section{Overview of IEEE 802.15.4 Protocol}

IEEE 802.15.4 is the emerging next generation standard designed for low-rate wireless personal area networks (LR-WPAN) [7]. IEEE 802.15.4 protocol is a standard for low rate-wireless personal area network (LRWPAN). It consists of low cost, less complex, low energy consumption, star topology, and also peer-to-peer is another particular things of this standard [8]. Physical layer of this standard is 868/915 MH, or almost $2.4 \mathrm{GH}$. MAC layer uses from CSMA-CA mechanism. It is also designed, for WSNs applications that need low range communication with maximum battery life time, it allows star topology, and point to point topology to be formed between networks [9] [10]. ZigBee technology are created satisfy market need for its low price with low potential, and low rate for WSNs. This technology is formed based on IEEE 802.15.4 standard.

It defines layers of PHY, and MAC to transfer data with low rate in (LR-WPANS) low rate wireless personal area network [9]. IEEE 802.15.4 standard is also known as of wireless personal network with low rate (LRWPAN). It is wireless communication network with low range, and less complicated, are used for application programs, with limit potential, and it has been designed for fixed necessity, efficiency. On the other hand 802.15.4 standard protocol doesn't privately defind for sensor networks, but it is not the same for WSNs. This standard is part of WPAN family, usually has a 10 meters of Personal Operating Space (POS). The sensor

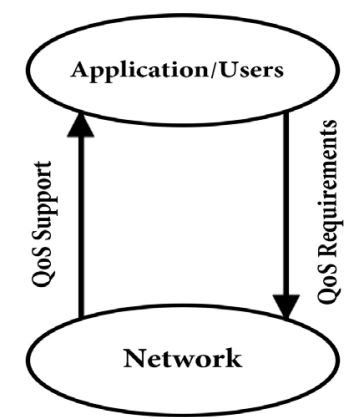

Figure 1. A simplified QoS model redrawn from [6]. 
network community has a strong background to attract in the use of these protocols for sensor networks. Recent studies have shown IEEE 802.15.4 MAC protocol in terms of efficiency, reliability, and energy (if otherwise proper parameter setting) may be severely limited. Hence, for an optimal parameter setting for reliability and minimal power consumption provide some suggested solutions. IEEE 802.15.4 systems are designed to support physical layer and link layer protocols. Physical layer of low bandwidth 915.868 Hrz, 2.4 G. Hrz high bandwidth. Radio channel access control can be used by MAC layer [9].

\section{Overview of LEACH Protocol}

WSNs consists of Base Stations (BS) and several nodes which are unattended and have limited resource and they can work until the energy exists inside them [11]. Low energy adaptive clustering hierarchical (LEACH) is the first network protocol that uses hierarchical routing for WSNs to increase the life time of network. LEACH is a cluster based protocol. Performance of LEACH protocol evaluated in NS2 simulator by following [11]: 1) throughput; 2) energy of cluster heads; 3) packet delivery ratio; 4) end-to-end delay.

Among communication protocols presented, LEACH protocols are particular importance to researchers for the following reason. First, a cluster networks are formed randomly, adaptively, and self-configured.

These properties are described as follows.

\subsection{Randomly}

This means that in each period, a certain number of nodes are randomly selected as a cluster head, and certain nodes have already been considered as cluster heads. The advantage of this feature is low over head of selecting the cluster head.

\subsection{Adaptively}

Nodes in each round, a certain number of randomly self generated nodes are introduced as the cluster head, the next round will not be able to take on the role of candidate. Thus, in each period, according to the cluster head candidates are identified earlier. Thus it expected that at the end of a certain number of periods, all nodes are clustered as supervisor.

\subsection{Self-Configured}

In this protocol, nodes are formed without the aid of any external factor or certain nodes in the network, and this helps the scalability of the protocol. The second importance of transferring data from the nodes of the cluster in the LEACH the cluster head to the sink station will be done by local control, and do not need the help of external factor, or certain nodes in the network to transfer data. As it was mentioned previously, LEACH uses the combination of data from each cluster, and transfers compacted data to the base station. Third stage, MAC protocol was used in LEACH, giving rest to nodes, save energy on consumption. As mentioned earlier, LEACH by a combination of data and transferring compacted data to base station. Therefore, number of sent and received will be reduced on the network, and redundant data due to the proximity sensor together are moved before posting. The kind of combination of data in LEACH is not fixed and depends on the wireless sensor application in networks. The purpose of this protocol is to balance the energy consumption in nodes. Classic options such as DT, MTE energy balance between the nodes are not guaranteed. The MTE leads to the lowest cost path. Where the measure of the cost is consumption power. Because nodes closer to sink, transferring of further data of nodes, there for it dies sooner. Thus a large amount of environment in a long time of network life cannot be monitored. One solution is the use of LEACH protocol, distributes the energy with a dynamic selection of clusters and clustering. This is the way sensors are divided into sections that each section has one cluster head and after an event sensors of each zone, send their data to cluster head. Cluster head sends these data directly. LEACH is the first protocol for hierarchical routing in sensor networks, and it is the base for most of the hierarchical protocols. On hierarchical protocols, performance of sensors are different. These means duties of some of the sensors are the collection of data, and some of them send data. In this protocol duty of transferring data is Cluster Heads (CH's) jobs, because they had higher energies than the other sensors. LEACH protocol has two main phases: 1) set up phase, 2) steady-state phase.

In each phase, implementation of these two places are called a process. On the first phase, cluster heads are 
selected, and then cluster members. Sensor $n$ produces a random number between zero, and one. If this number is less than $T(n)$, sensor $n$ is selected as cluster head. $T(n)$ is calculated as [1]:

$$
T(n)= \begin{cases}\frac{P}{1-P[r \bmod (1 / P)]} & \text { if } n \in G, \\ 0 & \text { otherwise, }\end{cases}
$$

where

$G=$ Group of nodes not selected as CHs in preceding $1 / p$ rounds;

$P=$ Recommended percentage of $\mathrm{CH}$;

$r=$ Current round.

When cluster heads are selected, each one sends a message to introduce itself as cluster head in network. And sensors according to the power of signals from cluster heads, choose the nearest cluster heads, then cluster head by running a time programming (TDMA), gives a time period limit to each node to send received data. In the second phase, node cluster head send data from environment to cluster head as scheduled (TDMA). Cluster head before sending data to base station, avoids from sending (Etra) data, process over received signals.

\section{Overview of CBRP Protocol}

On the other hand Cluster Based Routing Protocol (CBRP) is a robust/scalable routing protocol for Mobile Ad-hoc Networks (MANETs) superior to the existing. Since CBRP is a robust and scalable routing protocol for ad-hoc, CBRP is used as a routing protocol in our simulations. In this routing protocol all nodes divided into some clusters. Any cluster node connects to other clusters by cluster head via inter-links between them. To reduce energy consumption in this type of network we put all idle member nodes except cluster heads and gateways nodes to sleep mode. Our experiments show that the energy consumption is significantly reduced but to have the maximum saving energy we must pay its expenses by packet delivery delay. CBRP is a robust/scalable routing protocol for MANETs and superior to the existing methods [12] (e.g. the overhead of it is less than and throughput of it is more than of AODV) [3]. CBRP is a routing protocol designed for medium to large mobile ad-hoc networks. The protocol divides the nodes of the ad-hoc network into a number of overlapping or disjoint 2-hop diameter clusters in a distributed manner. Each cluster chooses a head to retain cluster membership information. The algorithm is a variation of the "lowest ID" cluster algorithm. The node with a lowest ID among its neighbors is elected as a Cluster Head $(\mathrm{CH})$. Each node maintains a neighbor table and a cluster adjacency table. Neighbor table is a conceptual data structure that it employs for link status sensing and cluster formation. Cluster adjacency table keeps information about adjacent clusters for adjacent cluster discovery. These tables are updated by the periodic Hello Messages (HM) [12].

\section{Related Works}

A WSNs contains hundreds or thousands of these sensor nodes. These sensors have the ability to communicate either among each other or directly to an external Base-Station (BS). Sensor nodes can be used to collect information from the environment, locally process this data and transmit the sensed data back to the user. Basically, each sensor node comprises sensing, processing, transmission, mobilize, position finding system, and power units. These nodes collect and transmit the information [9]. On reference [13], QoS are in 3 different groups: quality of end-to-end service (achieve the speed or accuracy end-to-end transmission) reliability assurance (ensure packets reach destination) an depends on the application (number of active nodes).

On reference [14] two parameters of data transfer and energy consumption of a communication channel in sensor network is considered as a base for quality service. On reference [15] multiple routing methods are introduced as a technique to improve QoS in WSNs. Multiple routing methods by creating several multiple routes between the source and destination of nodes improve criteria such as reliability, power consumption, delay, throughput and bandwidth. On reference [16] analysis of IEEE 802.15.4 ZigBee performance for star topology with the traffic scenarios CBR, FTP with simulation using NS. Packet delivery rate is 100 percents on a star topology with CBR traffic compared to the other traffics. Because with CBR traffic there is no acknowledgement for control. Lost packet are less on CBR, because it provides constant bit rate during the simulation on general factor of traffic load on CBR traffic is larger. In this article [16] to improve the performance of star topology for programming of simulation scenarios like sensors in WSNs to a control central nodes, monitoring of systems in 
the future are suggested. On reference [17] it is shown: one of the important quality service evaluation is the number of the sender, or active nodes in the network. According to increase of sensors, by activate of a number of sensors, and disactivate the rest of them, same time improving the QoS requirements, increase the lifetime of the network. Active or deactive method of sensors have a direct effect over the lifetime of those, and network. Because giving a method that use equal energy of all nodes is very effective to increase network lifetime. This study presents a method based on sensors energy to control service quality. Comparing this method with similar methods is shown that using this method not only QoS increase, but was also increased life time of the network very much. On reference [18] several QoS-aware protocols are introduced. These protocol minimize the bandwidth usage and delay. Also choose the routes with minimum expenses to communication, assure to transfer packet. Thus the service quality is very important according to the kind of application in WSNs is very different, comparing to the old networks. Therefore research on efficiency of standard protocols, and base for satisfying service quality are necessary to improve performance of the network. An improved and more efficient protocol for WSNs in order to provide QoS and improve service parameters can enhance the performance and efficiency of the network. In this context, improving service quality, schedule, and cost and time spend by type of program in WSNs as the optimal can increase the lifetime of network. Since a variety of applications in WSNs need to provide different quality parameters such as delay, reliability, amount of bandwidth, etc. Design of a protocol due to the problems caused by the unique characteristics of WSNs such as the limited bandwidth, unreliable communication, vulnerability of nodes and more. Requirements are considered essential for QoS in networks. The result of this research can provide guidance and direction to research and solve problems related to QoS requirements, and help to achieve the required QoS in WSNs.

\section{Existing Requirements \& Challenges for QoS in WSN}

As defined in [19] [20], Quality of Service is a set of service requirements to be met by the network while transporting a flow. "Here a flow is" a packet stream from source to a destination (unicast or multicast) with an associated (QoS) [19]. In other words, QoS is a measurable level of service delivered to network users, which can be characterized by packet loss probability, available bandwidth, end-to-end delay, etc. In this section, first, we have discussed about the QoS requirements \& challenges in WSNs followed by the major existing approaches for supporting QoS in WSNs. The requirement of QoS in WSNs can be specified from two perspectives [13]. These are application specific QoS and Network QoS. As discussed in Section 1, QoS parameters in WSNs may vary depending on the application domain. Some of the application specific QoS parameters are data accuracy, aggregation delay, fault tolerance, coverage [21], optimum number of active sensors [22] etc. The application demands certain requirements of the deployment of sensors which are directly related to the quality of application. From the network perspective, it has been considered as how to provide QoS constrained sensor data while optimally utilizing sensor resources. Every class of application has some common requirements in network. The network is concerned with how to transmit the sensed data from the sensor field to the sink node fulfilling the required QoS. There are three data delivery models in sensor network [23]. These are event driven, query driven and continuous. The event driven application in WSNs is mostly delay tolerant, interactive and non end-to-end. The sensors detect the occurrence of certain events and to take action accordingly. In one side of the application there is a sink node and on the other side a group of sensor nodes which are affected by certain events [6]. The query driven application WSNs are interactive, query based, delay tolerant, mission critical and non end-to-end. The queries are generated by the sink node on demand and sent to sensor nodes inquiring occurrence of certain events. In traditional data network, QoS defines certain parameters such as packet loss, delay, jitter, bandwidth etc. However, the QoS requirements in WSNs such as data accuracy, aggregation delay, coverage, fault tolerance and network lifetime etc.

\section{Simulation Setup and Performance Metrics Analysis}

Analysis, giving an opportunity to specify the ideal and the maximum number of simulations. In this section, we present our simulation setup for 802.15.4, LEACH and CBRP protocol, followed by the performance metrics and comparisons. The main goal of simulation is to analyze the performance of different routing. We choose to simulate the NS 2 simulator to make it more flexible and has better performance. In this section we simulate and implement the three protocols. Simulation was performed with the software simulator NS 2.34, NS 2.29 on Linux. Values of the simulation parameters are given in Table 1. Simulation time is 140s. We achieve values of parameters of QoS at $40 \mathrm{~s}$ up to $140 \mathrm{~s}$ degrees, and then compare those with each other. These values are calcu- 
Table 1. The traffic and node parameters.

\begin{tabular}{cccc}
\hline Parameters & Values & Parameters & Values \\
\hline Simulation area & $100 \mathrm{~m} \times 100 \mathrm{~m}$ & Bas station & $(0,0) \mathrm{m}$ \\
Number of nodes & 200 & Data payload & 512 bytes/packet \\
Max queue size & 200 & Routing & AODV \\
Traffic type & CBR (UDP) & Queue size & 200 \\
Queue type & Drop Taile & Antenna type & Omni directional \\
Initialise energy & $0.5 \mathrm{j}$ & Chanal type & Wireless channel \\
Radio propagation model & Propagation/TwoRayGround & Link type & LL \\
Simulator & NS 2.29 and 2.34 & Simulation duration (sec) & $40,60,80,100,120,140$ \\
Transmission range & $10 \mathrm{~m}$ & MAC layer & LEACH, 802.4.15, CBRP \\
Packet rate & 4 packets/sec & Movement & Chain topology \\
\hline
\end{tabular}

lated using $\mathrm{C}++$ codes. The simulation scenario is obtained and discussed according to the simulation settings (Table 1). In the simulation environment, the sensor was considered $100 \times 100 \mathrm{~m}$. Assume that the range of the sensor is 20 meters. In addition the initial energy of each sensor node was chosen 0.5 jules. Test for the number of sensor $\mathrm{N}$ nodes were performed, was equal to 200. Since the most important for evaluating the effectiveness are the quality of services (compare function) of protocols (Figure 2). 8-bit benchmarks for QoS parameters are considered to compare the performances of three protocols. Then, using scenarios, we analyze separately the parameters of QoS. The results obtained were compared with each other.

\section{Result and Discussion}

Performance of 802.15.4, LEACH and CBRP is evaluated with the help of AODV. The performance parameters considered in our experiments include the Throughput, Average HOP, Normalized Routing Load, Average Delay, Number of Packets Loss, Packet Delivery Ratio, Average Data Delivery, Delay. It should be noted that parameter values are calculated based on the simulation time, not based on the number of packets sent or received. Performance evaluation matrices for all above protocol is given below:

\subsection{Scenario 1: Throughput}

The effective number of data current at a certain time of transfer are called throughput. This mean to total of packets received at the destination in a node is called throughput. These parameters are used as the bandwidth in some situations. In general, larger throughput network, performance is better. In Figure 3 received packets in destination node are shown for two protocols. On Figure 3 are shown standard protocol 802.15.4 at the time 40 s during the simulation, and also when running, and end of the simulation have more throughput that LEACH protocol, and increases linearly.

\subsection{Scenario 2: Average HOP}

On Figure 4 it is shown 80.15.4 protocol has used more number of steps to send packet to destination. Almost it has been fixed during the simulation time. While the LEACH protocol during the times $40 \mathrm{~s}$ up to $140 \mathrm{~s}$ stimulation have used less steps. This caused less traffics, at the result less delay in the internet.

\subsection{Scenario 3: Normalized Routing Load}

As in Figure 5 it is shown this parameter on LEACH protocol during simulation had a better condition. It means Network during simulation has no jump. In this case also LEACH protocol, has present higher QoS.

\subsection{Scenario 4: Average Data Delivery Delay}

The delay metric between two nodes represented as is the sum of the processing, queuing, transmission, and 


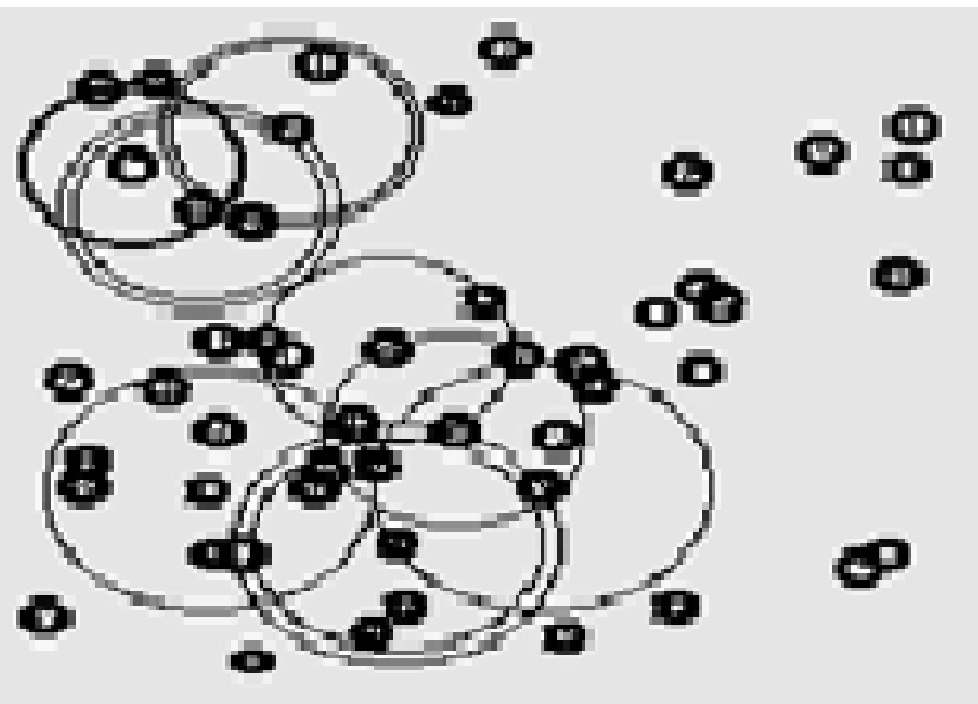

Figure 2. Simulation model.

$\longrightarrow$ LEACH $\rightarrow-802.15 .4$

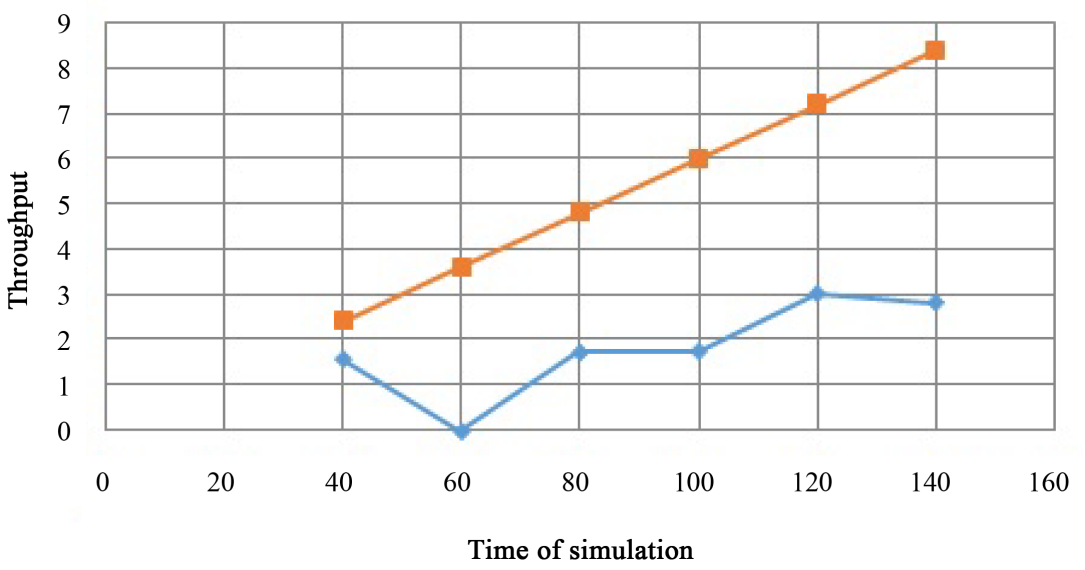

Figure 3. Comparison of throughput for 802.15.4, LEACH.

$$
\rightarrow-\mathrm{LEACH} \rightarrow-802.15 .4
$$

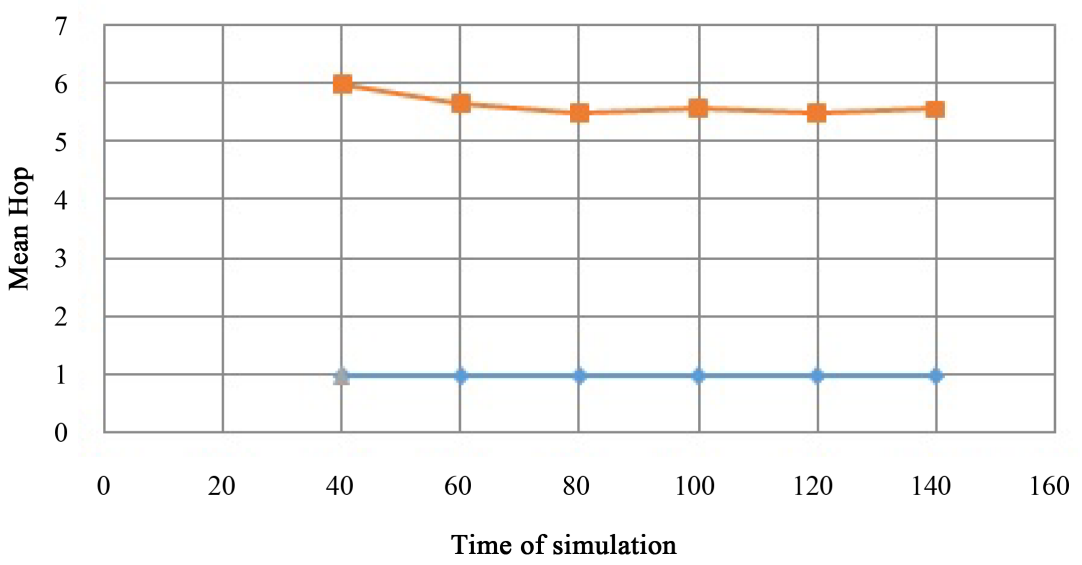

Figure 4. Comparison of HOP average for 802.15, LEACH. 


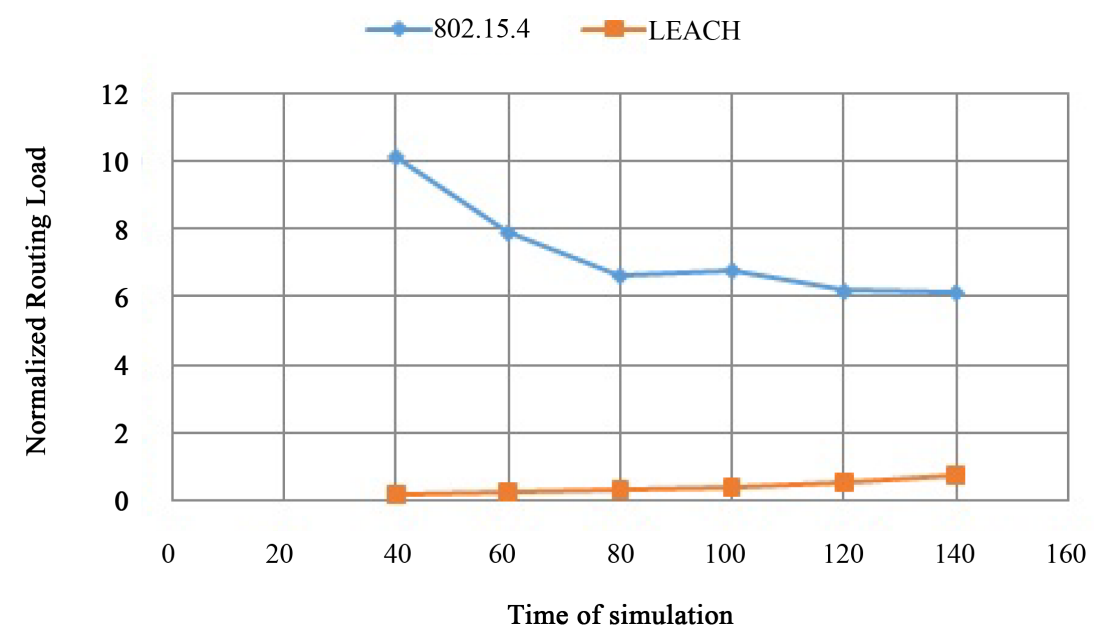

Figure 5. Comparison of normalized for 802.15.4, LEACH.

propagation delay [14]. The average data delivery delay is the end-to-end delay experienced by successfully received packets [24]. On Figure 6 it shows 802.15.4 at time $40 \mathrm{~s}$ during running of simulation has had less average delay time than Leach protocol. While 802.15.4 protocol increased, and had less fluctuations. In this case also 802.15.4 protocol presented higher QoS. So, average delay on sending packets using 802.15.4 standard protocol in this simulation is less than the LEACH protocol, and it has shown better performance. Therefore delay happens, but the amount of delay in the network as of parameter of quality service with 802.15.4 standard protocol will be obvious. In this case also 802.15.4 has presented higher QoS.

\subsection{Scenario 5: Number of Packet Loss}

The simulation is carried out with 200 active nodes. One of them being the coordinator. The nodes remain stationary. Figure 7 below represents number of all loss packets by all the 200 nodes in the network. On Figure 7 it is shown that 802.15.4 standard protocol at the time $40 \mathrm{~s}$ when running simulation has lost less packets than LEACH protocol in the network. Also continue to be run simulation amount of lost packets in the network had less increased at the end of $140 \mathrm{~s}$. While, LEACH protocol such a linear has lost more packets. Therefore according to the rate of sending packets are the same for both protocols, but amount of lost packets in whole network during simulation are more on LEACH protocol. This amount shows the high efficiency of 802.15.4 protocol to the LEACH protocol. In this case also 802.15.4 protocol, has shown better QoS. Also, it shows the effect of the AODV protocol which ensures the efficiency.

\subsection{Scenario 6: Packet Deliver Ratio (PDR)}

The packet delivery ratio is one of the most important metrics in real-time applications which indicates the number of packets that could meet the specified QoS level [24]. It is the ratio of successful packet receptions referred to as received packets, to the attempted packet transmissions referred to as sent packets [24]. Whatever the rate of delivery of packets be higher, performance of the network is good, and it means the performance of protocol in the network is better, if it is low, performance is not good.

In Figure 8 it has shown, delivery rate of healty packets (or number of packets arrived by all nodes) during time $40 \mathrm{~s}$ up to $140 \mathrm{~s}$ for 802.15 .4 standard protocol was fixed (was constant). While the LEACH protocol was decreased such linear, and had no fixed rate (constant rate). In this case also 802.15.4 protocol has shown higher QoS.

\subsection{Scenario 7: Drop Packet}

In Figure 9 it shows 802.15 .4 standard protocol at the time $40 \mathrm{~s}$ simulation of packets dropped less than LEACH protocol. Also to continue to be run simulation of packets in the network at the end of time $140 \mathrm{~s}$, had dropped less. While LEACH protocol at end of $140 \mathrm{~s}$ almost had dropped packet about 3 times of 802.15.4 protocol. 


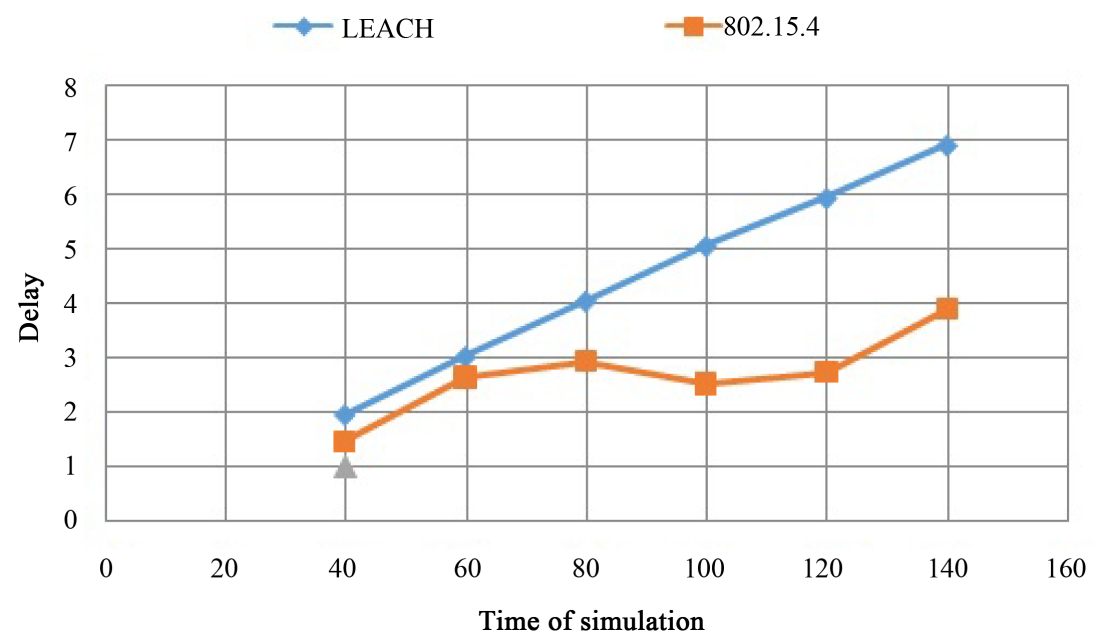

Figure 6. Comparison of average delay for 802.15.4, LEACH.

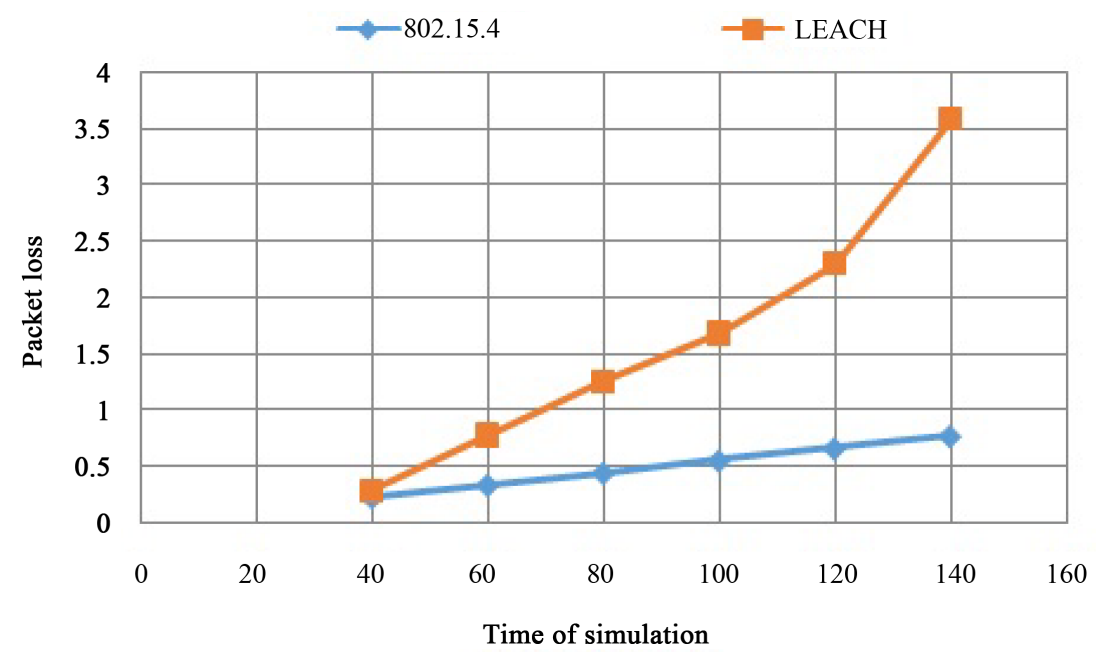

Figure 7. Comparison of number of loss packets for 802.15.4 and LEACH.
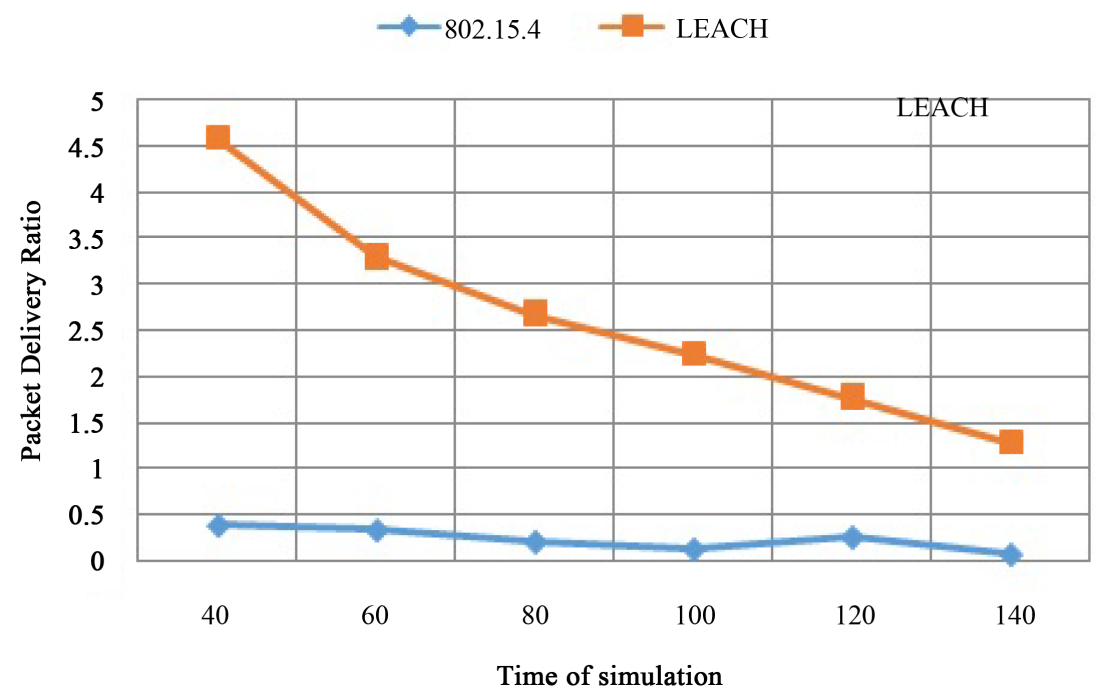

Figure 8. Comparison of packet delivery rate for 802.15.4 and LEACH. 


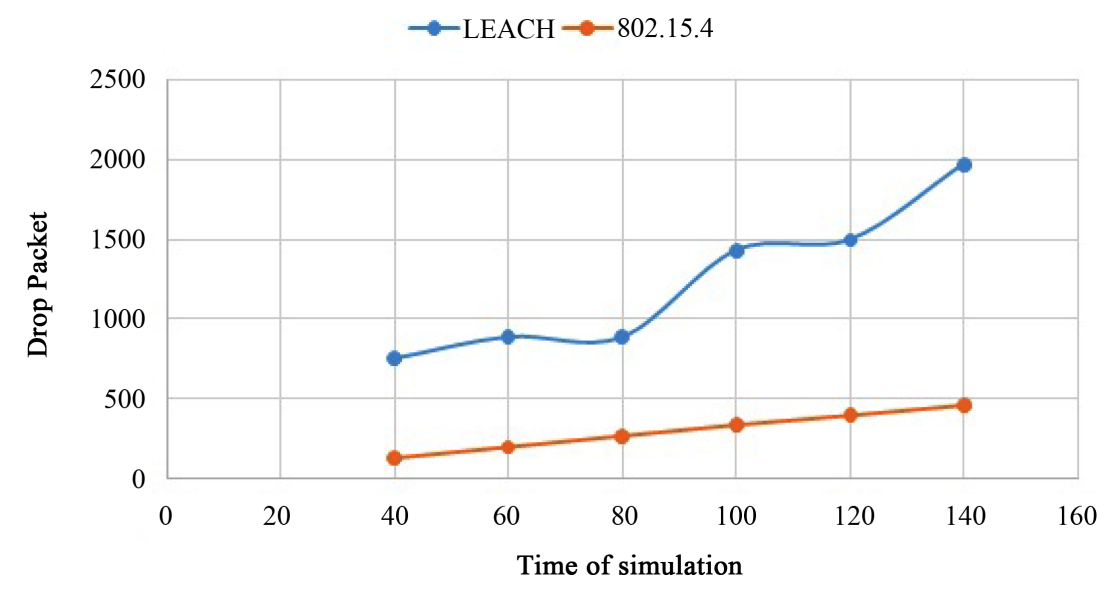

Figure 9. Comparison of drop packet for 802.15.4 and LEACH.

Therefore the amount of dropped packets on all the networks during simulation make obvious higher performance more than 802.15.4 protocol with respect to LEACH protocol. In this case also 802.15.4 protocol has presented higher QoS.

\subsection{Experiment 8: Energy Consumption}

As, a certain number of nodes are selected to transmit results to the gateway, the network might consume energy differently depending on the network topology and the number of information transmitting nodes. The average energy consumed is an indication of the energy consumption in transmission and reception of all packets in the network. This metric reveals the efficiency of an approach with respect to the life time of a WSNs [24].

The average energy consumption per transmission is the index of the network lifetime; less energy consumption per transmission indicates more network lifetime. Network lifetime is given in terms of when the energy of a first node drops under the energy threshold [25]. On Figure 10 we can see 802.15.4 standard protocol between time $40 \mathrm{~s}$ up to $140 \mathrm{~s}$ during the period of simulation in average has used less energy than LEACH protocol, and energy consumption of that was improved. Also as it has shown a large amount of energy was saved with nodes. So lifetime of 802.15.4 protocol, average more than LEACH protocol. Therefore performance of 802.15.4 protocol to the energy consumption is more than LEACH protocol. In this case also 802.15.4 protocol has present higher QoS.

\section{Comparison of Performance Evaluation 802.15.4 and LEACH with CBRP}

In this section CBRP protocol by using simulated NS 2.34, and QoS parameters values were compared with previous scenarios. By looking at Figure 11 where rate of delivery of healty packets during the times $40 \mathrm{~s}$ up to $140 \mathrm{~s}$ for 802.15.4 protocol was fixed (constant), but on LEACH protocol like linear was decreased, and has no fixed rate (constant rate). Also CBRP algorithm with packet concentration were decreased, and it cause to increase the healty packets rate on destination. Here we see LEACH protocol has a weak signal on receiving healthy packets. CBRP protocol has a high power point. Also 802.15.4 protocol has a fix (constant) rate.

In Figure 12 we see 802.15.4 standard protocol at time $40 \mathrm{~s}$ was running simulation has lost less packets than the LEACH protocol in the network. Also the amount of lost packets in the network at the end of time $140 \mathrm{~s}$ less increased during running simulation. While the LEACH protocol such linear has lost more packets. So the amount of lost packets in all networks during simulation, also had better performance than LEACH protocol. Also algorithm CBRP by reducing the packet density and the overhead can cause reduced lost of the packets. Here, it showed that weak point of LEACH protocol about losing packets, is more clear, and obvious.

According to Figure 13 it shows 82.15 .4 protocol at the time $40 \mathrm{~s}$ on running stimulation, also during running, and end of stimulation has had higher throughput than Leach protocol. Also CBRP algorithms with decrease on packet density, and overhead cause higher throughput. In this case also 802.15.4 protocol has presented higher QoS. CBRP protocol has a strength point. Also, strength point of 802.15.4 protocol is higher than LEACH protocol. 


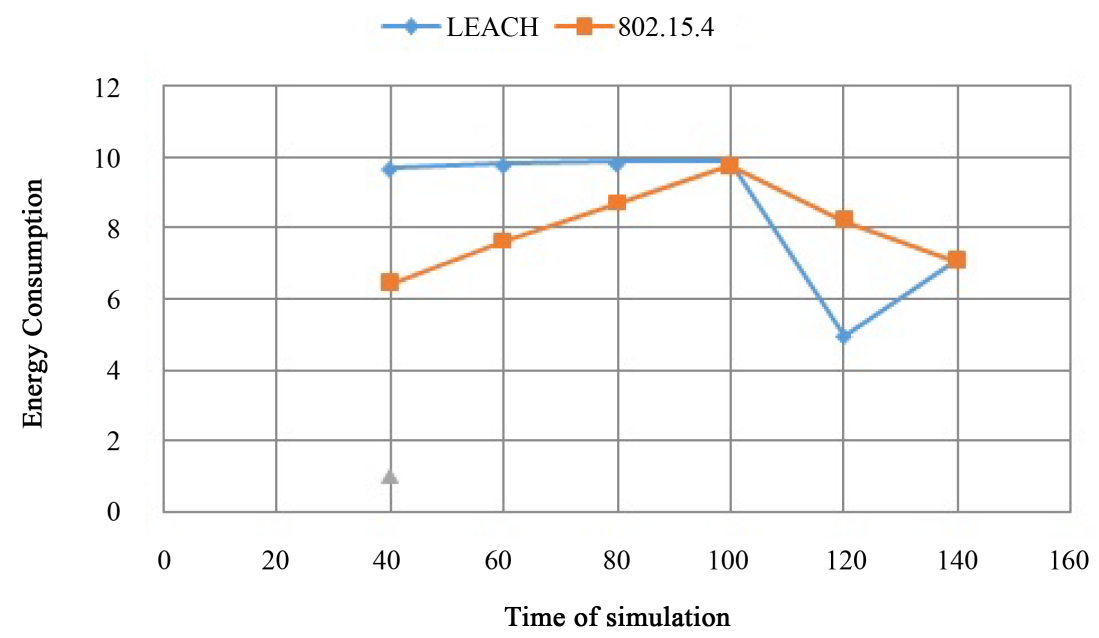

Figure 10. Comparison of energy consumption for 802.15.4 and LEACH.

$\longrightarrow$-802.15.4 $\rightarrow$ LEACH $\longrightarrow$ CBRP

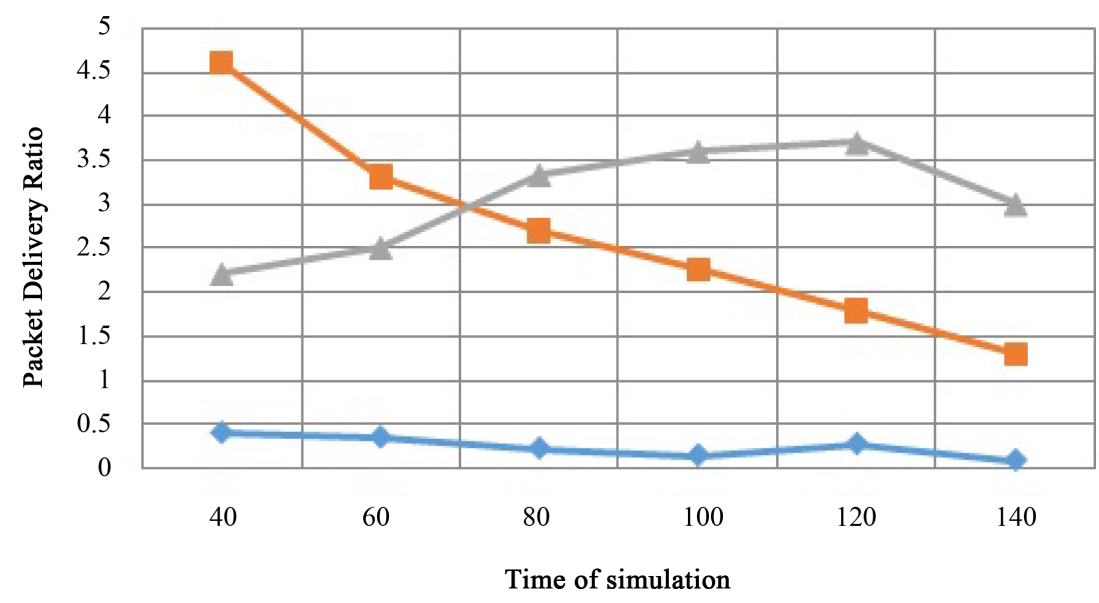

Figure 11. Comparison of packet deliver ratio for 802.15.4, LEACH and CBRP.
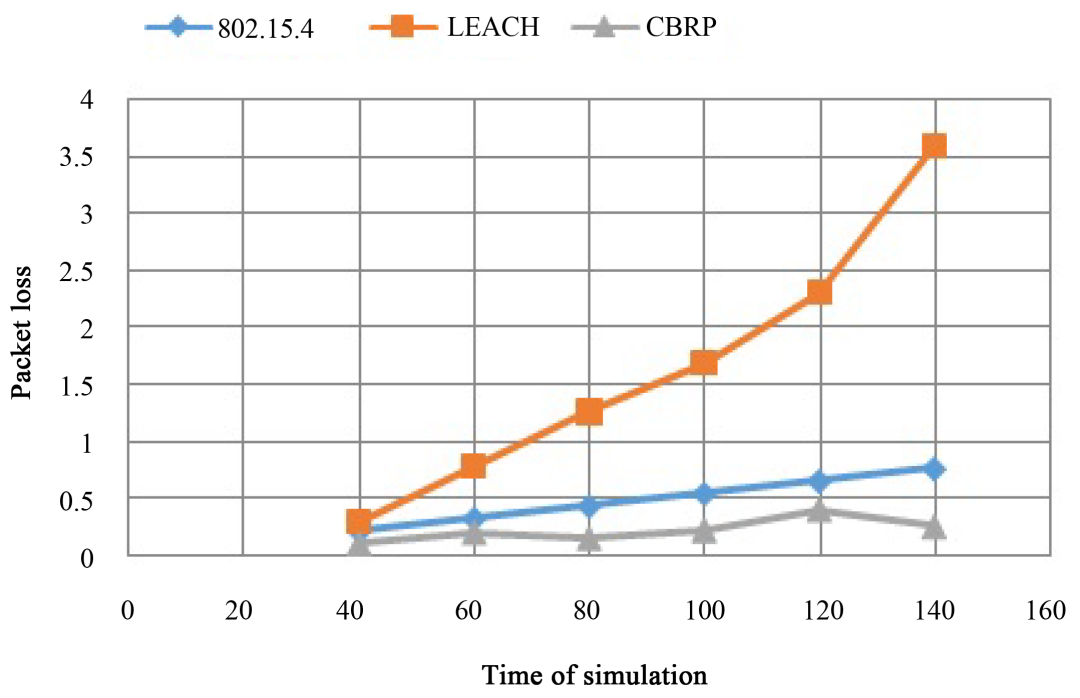

Figure 12. Comparison of packet loss for 802.15.4, LEACH and CBRP. 


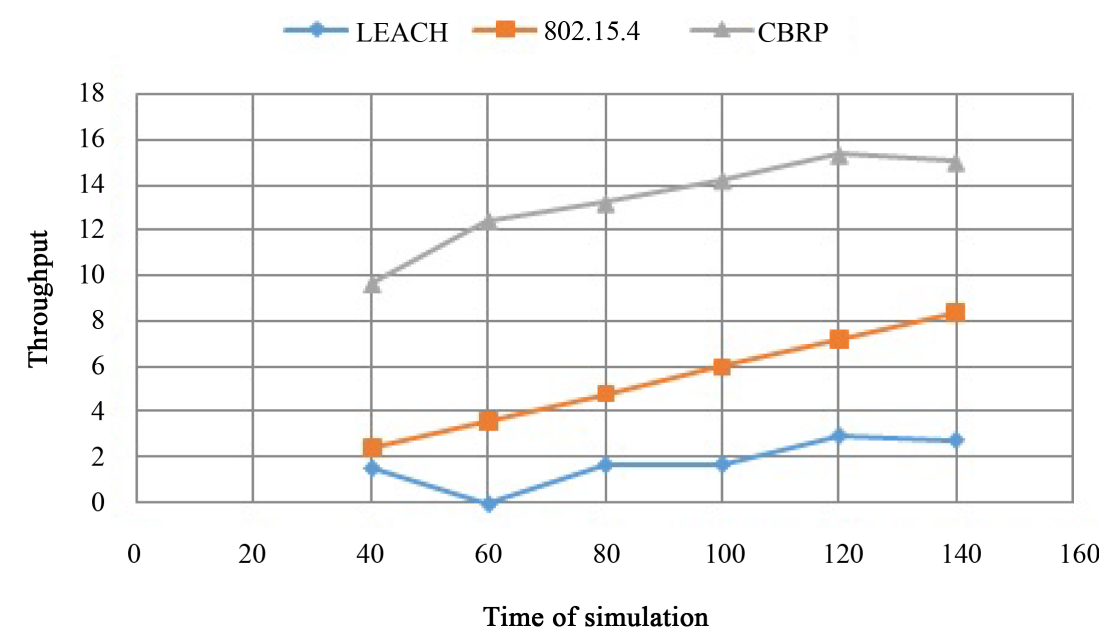

Figure 13. Comparison of throughput for 802.15.4, LEACH and CBRP.

On Figure 14 we see 802.15.4 standard protocol at the time $40 \mathrm{~s}$ simulations of packets decreased less than LEACH protocol. Also at the end of time $140 \mathrm{~s}$ decrease of packets in the network were seen. While LEACH protocol at the end of time $140 \mathrm{~s}$ almost were lost (decreased) 3 times less than 802.15 .4 protocol on packets. The low rate of packet lost in the network during a simulation, the performance of the 802.15.4 protocol reveals with respect to the LEACH protocol. Also with CBRP algorithm because of the low density of packet, and reduce of the overhead amount of decrease of packets are less on both protocols. Here, the LEACH protocol is more weak than two other protocols.

On Figure 15 we see LEACH protocol used least number of steps to send packets to the destination. Almost during the simulation time it was constant. But 802.15.4 protocol during time of $40 \mathrm{~s}$ up to $140 \mathrm{~s}$ simulation has used more steps. This parameter causes more traffic, and at the result had more delay in the network. But the CBRP algorithm procedure causes decrease of overhead, and traffic package. As a result number of steps are less with respect to 802.15.4 protocol for sending packets. Here 802.15.4 protocol has a weakness, and to send the packet to the central nodes uses more steps. LEACH protocol has more power and almost is fixed (constant) during the simulation.

In Figure 16 it is seen 802.15 .4 standard protocol during times $40 \mathrm{~s}$ up to $140 \mathrm{~s}$ during the period of simulation average energy usage were less than LEACH protocol, and energy consumption was optimized. Also as you can see in Figure 16, more amount of energy is saved in nodes.

So the lifetime of network with 802.15.4 protocol is more than LEACH protocol. Therefore 802.15.4 protocol performance according to usable energy is more than LEACH protocol. Also with CBRP algorithms amount of energy consumption at the beginning, simulations, were less, and at last were more with respect to the other two protocols. Here CBRP had higher weakness, and during the simulation amount of energy usage was increased. In Figure 17 normalized parameter indicates the rate of fluctuations, changes, and jump in the network. This parameter on LEACH and CBRP protocols during time of simulation shows better conditions. 802.15.4 shows very high weakness, it means network during simulation has no jump, also fluctuation rates, changes, and jump with CBRP algorithms on the standard situation.

On Figure 18 it shows 802.15.4 standard protocol at the time $40 \mathrm{~s}$ during simulation had less delay time than LEACH protocol, also running delay simulation of LEACH protocol linearly was increased, while 802.15.4 protocol increased, and had less fluctuations. The average of delay on sending packets using 802.15.4 standard protocol in this simulation was less than LEACH protocol, has shown better performance. Therefore delay will happens but amount of delay in the network as of quality service parameter with LEACH standard protocol is more obvious. Also with CBRP algorithm amount of delay at the begging was decreased, and at the end was increased, but average delay time with respect to standard protocol was higher, and was less than LEACH protocol. This delay shows a weak point of LEACH protocol, that by providing effective efficient energy for LEACH protocol, and increase of a lifetime in network laminated that. Here CBRP protocol, LEACH protocol has high weakness, and 802.15.4 protocol is in better state.

As shown in Figure 19: 


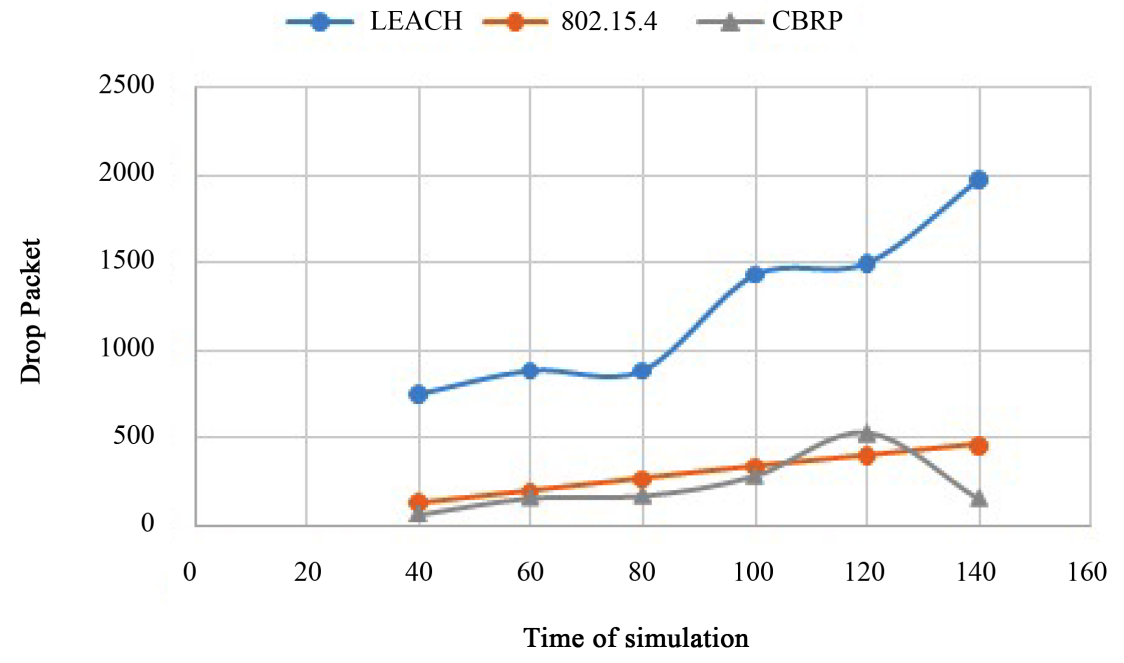

Figure 14. Comparison of drop packet for 802.15.4, LEACH and CBRP.

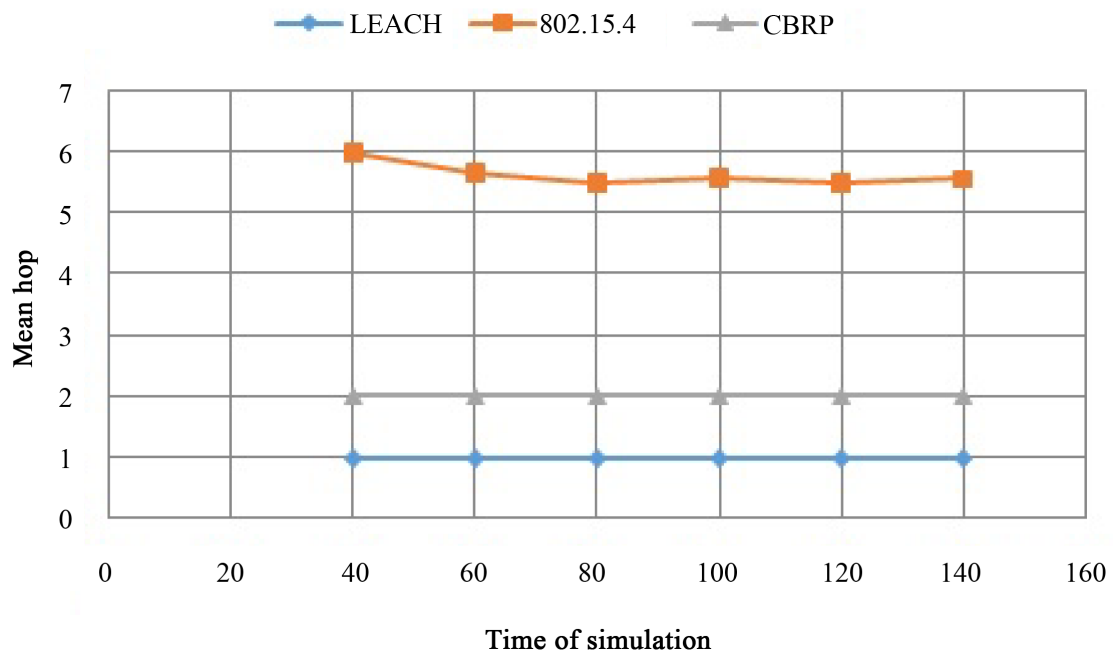

Figure 15. Comparison of mean HOP for 802.15.4, LEACH and CBRP.

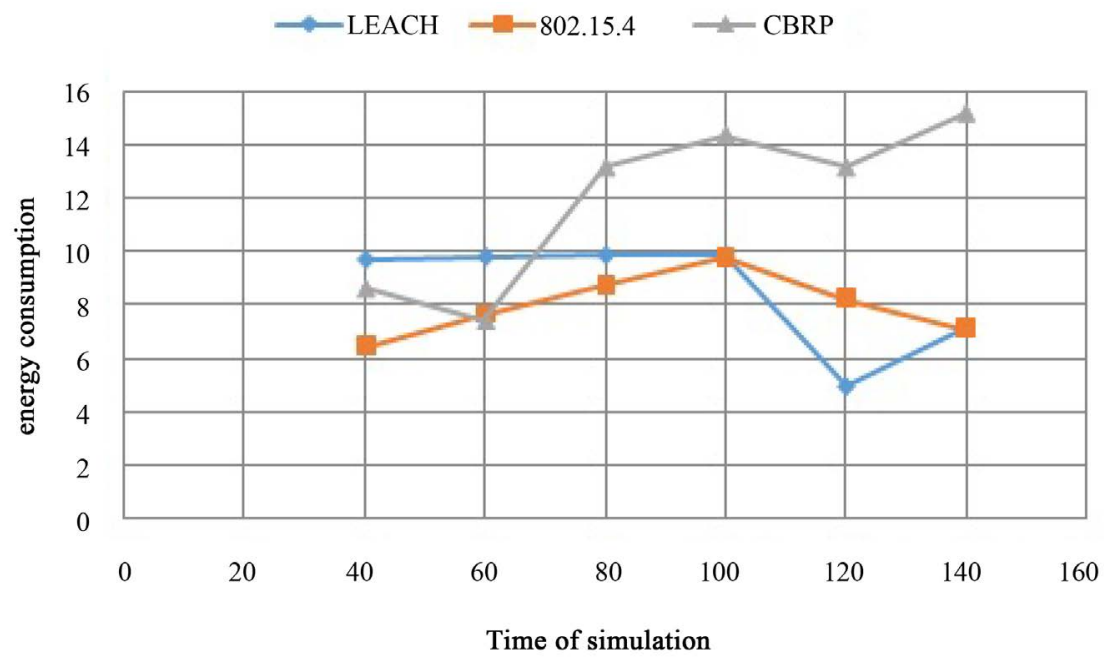

Figure 16. Comparison of energy consumption for 802.15.4, LEACH and CBRP. 
$\multimap$ 802.15.4 $\rightarrow$ LEACH $\rightarrow$ CBRP

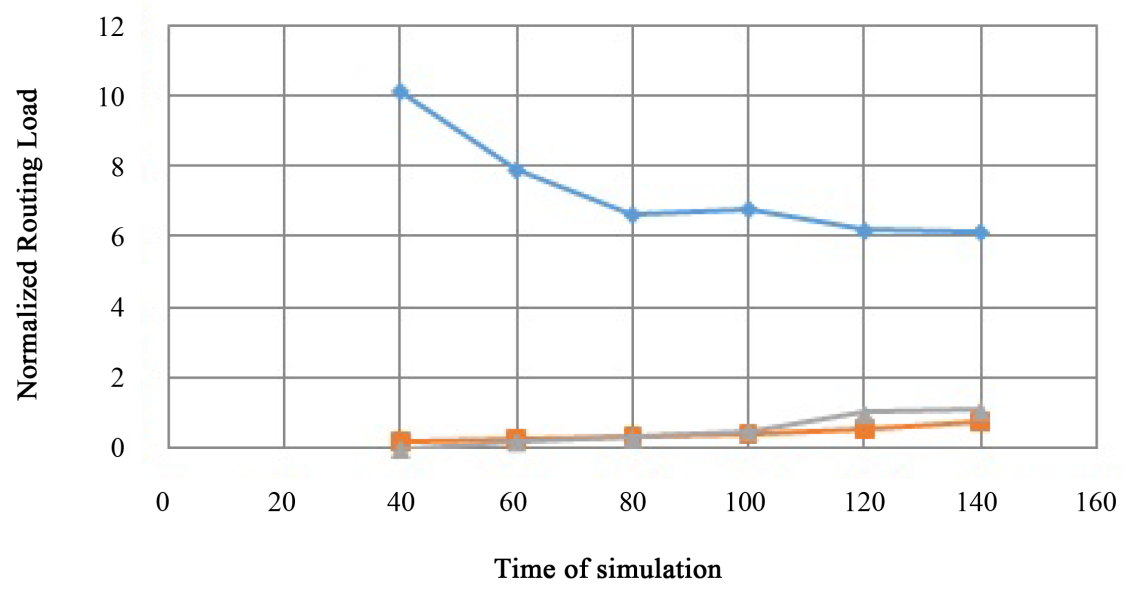

Figure 17. Comparison of normalized for 802.15.4, LEACH and CBRP.

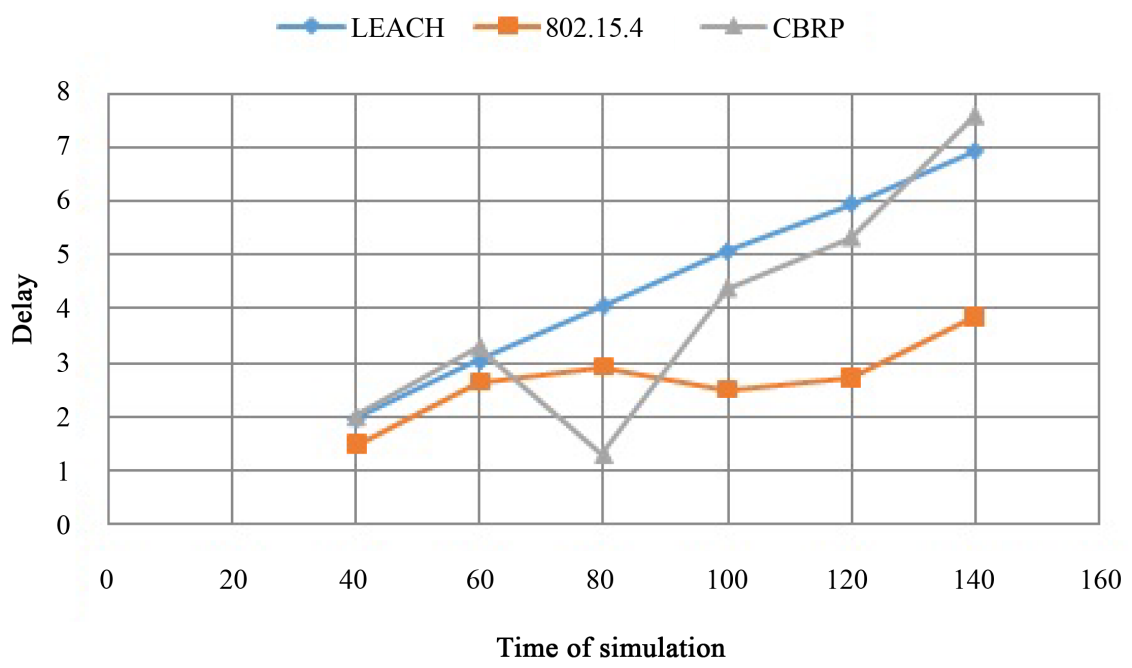

Figure 18. Comparison of average delay for 802.15.4, LEACH and CBRP.

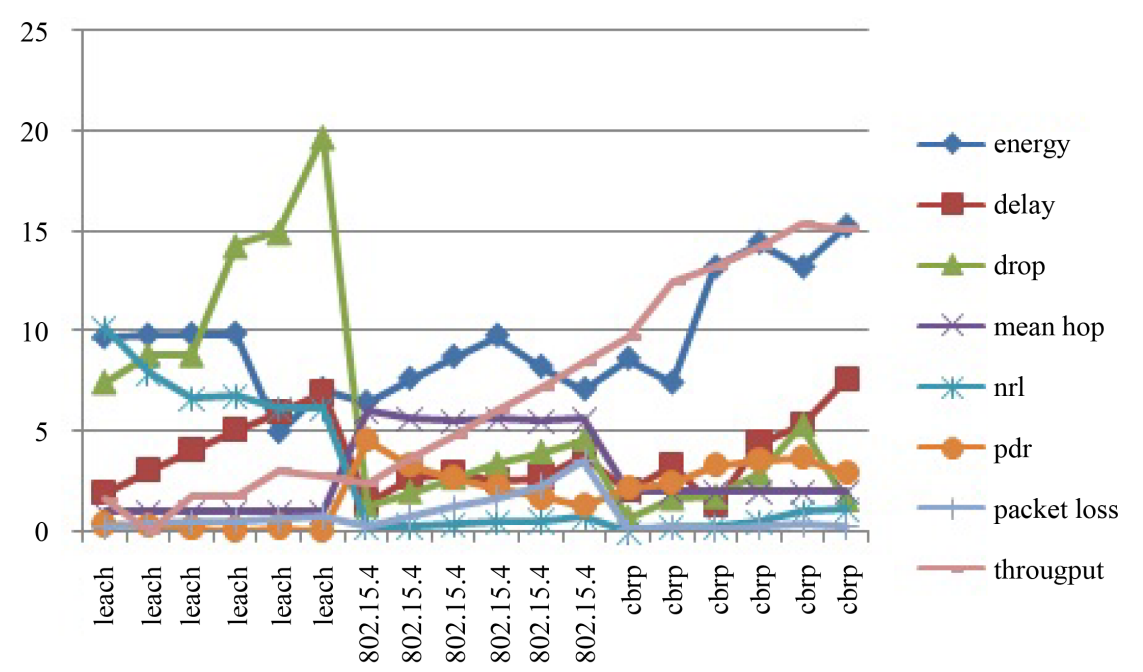

Figure 19. Comparison of QoS in 802.15.4, LEACH and CBRP. 
- In the case of lost packets the point of weakness of LEACH protocol, then the other two protocols.

- The parameter of the CBRP throughput protocol has higher strength.

- In the case of packets lost in the network, the LEACH protocol has more disadvantages than the other two protocols.

- In the case of mean HOP parameters of lost packet in the network, the LEACH protocol has more strength, that is fix during the simulation.

- In the case of parameter of energy consumption of weakness points CBRP protocol, it has higher during the simulation the energy consumption of it will increase.

- In the case of rate of fluctuations, changes, and jump in network 802.15.4 protocol has higher weakness.

- In the case of average delay of 802.15.4 protocol has better situation.

After analysis, evaluation of quality, and quantity of different service qualities on different conditions we came up with this conclusion that 802.15.4 is better than LEACH, and has higher efficiency, also CBRP is better than both of other protocols.

\section{Conclusion}

In this article, QoS parameters were studied in detail to evaluate the performance of three protocols. To do it, first three protocols 802.15.4, LEACH and CBRP were separately simulated in the NS simulator. Then we analyzed the simulation output. For evaluation of the performance of CBRP protocol, it used the NS 2.34. Simulation was done with a constant number of nodes in WSNs. The protocol used for the three protocols was ad-hoc on demand distance vector. QoS parameters were analyzed with following titles: average delay, throughput, energy consumption, number of packets lost, packet delivery ratio, normalization network and mean HOP. We were able to compare the protocol due to changes of the parameters of QoS. Also, the simulation results of CBRP algorithm were compared with other two protocols. Strengths and weaknesses of QoS parameters were known over the three protocols. Thus, by knowing the status of each one (each protocol) we can solve the weaknesses. About the packet delivery rate, LEACH protocol has a weakness. CBRP protocol has strengths. The 802.15.4 protocol has a fixed rate. At last, by analyzing all parameters of quality services, we got this conclusion that CBRP, route protocol for supplying service qualities is better and more efficient. Therefore it is more efficient and suitable to supply quality service. It has developed QoS more than the other two protocols. Also, using extensive simulation, we demonstrate the robustness of models and expand the initial work done in [1] [25] [26] on the above performance parameters. These include the assessment between multi-constrained QoS multipath routing in WSNs and the impact of AODV Protocol on the intensity of the network performance. Also, it shows the effect of the AODV protocol which ensures the efficiency.

\section{References}

[1] Akyildiz, I., Su, W., Sankarasubramaniam, Y. and Cayirci, E. (2002) A Survey on Sensor Networks. IEEE Communications Magazine, 40, 102-114. http://dx.doi.org/10.1109/MCOM.2002.1024422

[2] Brienza, S. (2013) Strategies for Optimal MAC Parameter Setting in IEEE 802.15.4 WSNs: A Performance Comparison, Computers and Communications (ISCC). 2013 IEEE Symposium on Computers and Communications (ISCC), Split, 7-10 July 2013, 000898-000903. http://dx.doi.org/10.1109/ISCC.2013.6755063

[3] Lotf, J.J. (2010) Quality of Service in Wireless Sensor Networks. The Third International Conference "Problems of Cybernetics and Informatics”, Baku, 6-8 September 2010.

[4] Chen, D. and Varshney, P. (2004) QoS Support in Wireless Sensor Network: A Survey. Proceeding of International Conference on Wireless Networks (ICWN 04), Las Vegas, 21-24 June 2004, 227-233.

[5] Ganz, A., Ganz, Z. and Wongthavarawat, K. (2004) Multimedia Wireless Networks: Technologies, Standards, and QoS. Prentice Hall, Upper Saddle River.

[6] Bhuyan, B., Sarma, D., Kumar, H., Kar, A. and Mall, R. (2010) Quality of Service (QoS) Provisions in Wireless Sensor Networks and Related Challenges. Wireless Sensor Network, 2, 861-868. http://dx.doi.org/10.4236/wsn.2010.211104

[7] Ali, K.A. and Mouftah, H.T. (2011) Wireless Personal Area Networks Architecture and Protocols for Multimedia Applications. Ad Hoc Networks, 9, 675-685. http://www.elsevier.com/locate/adhoc

[8] Mbowe, J.E. and Oreku, G.S. (2014) Quality of Service in Wireless Sensor Networks. Wireless Sensor Network, 6, 1926. http://dx.doi.org/10.4236/wsn.2014.62003 
[9] Rahmani, E. (2005) Design and Modelling of the Physical Layer Standard of IEEE 802.15.4. Panzdhymn Iranian Conference on Electrical Engineering, Iran Telecommunication Research Center, Tehran.

[10] Barai, L. and Gaikwad, M. (2014) Performance Evaluation of LEACH Protocol for Wireless Sensor Network. International Journal of Innovative Research in Advanced Engineering (IJIRAE), 1, 141-145. http://ijirae.com

[11] Hosseini-Seno, S.A., Wan, T.C. and Budiarto, R. (2009) Energy Efficient Cluster Based Routing Protocol for MANETs. Proceeding of International Conference on Telecom Technology and Application, Manila, 6-8 June 2009.

[12] Hosseini-Seno, S., Wan, T. and Budiarto, R. (2009) Energy Efficient Cluster Based Routing Protocol for MANETs. Proceeding of 2009 International Conference on Telecom Technology and Application Manila, Philippine, 6-8 June 2009, 435-436.

[13] Chen, D. and Varshney, P.K. (2004) QoS Support in Wireless Sensor Network: A Survey. Proceedings of the 2004 International Conference on Wireless Networks (ICWN2004), Las Vegas, 21-24 June 2004.

[14] Brienza, S. (2013) Strategies for Optimal MAC Parameter Setting in IEEE 802.15.4 Wireless Sensor Networks: A Performance Comparison. 2013 IEEE Symposium on Computers and Communications (ISCC), Split, 7-10 July 2013, 000898-000903. http://dx.doi.org/10.1109/ISCC.2013.6755063

[15] Meybodi, M. (2009) Quality Control Services Based on Wireless Sensor Networks Energy Sensor. 15th Annual International Conference of Computer Society of Iran, Tehran, 2009.

[16] Priyanka, S., Kumar, M., Jaiswal, A. and Saxena, R. (2013) Analysis of ZigBee (IEEE 802.15.4 Standard) for Star Topology with AODV Protocol. International Journal of Innovative Technology and Exploring Engineering (IJITEE), 3.

[17] Kauar, T., Paulus, R., Jaiswal, A.K. and Ashok, A. (2014) QoS Analysis in IEEE 802.15.4 and Impact of Flag Mobility on Network Performance in WSN. International Journal of Computer Applications, 90, 16-20.

[18] Kumar1, V.N., Sankar, K.S., Srinivasa Rao, L. and Santhi Prabha, I. (2012) Comparative Analysis of QoS-Aware Routing Protocols for Wireless Sensor Networks. Innovative Systems Design and Engineering, 3.

[19] Crawley, E., Nair, R., Rajagopalan, B. and Sandick, H. (1997) A Framework for QoS-Based Routing in the Internet. https://tools.ietf.org/html/rfc2386.html

[20] Wright, W. (1906) Flying-Machine. US Patent No. 821393.

[21] Iyer, R. and Kleinrock, L. (2003) QoS Control for Sensor Networks. IEEE International Conference on Communications, 1, 517-521.

[22] Tilak, S., Abu-Ghazaleh, N. and Heinzelman, W. (2002) A Taxonomy of Wireless Micro-Sensor Network Communication Models. ACM Mobile Computing and Communication Review (MC2R), 6, 28-36.

[23] Bagula, A. (2010) Modelling and Implementation of QoS in Wireless Sensor Networks: A Multiconstrained Traffic Engineering Model. EURASIP Journal on Wireless Communications and Networking, 2010, Article ID: 468737. http://dx.doi.org/10.1155/2010/468737

[24] Alwan, H. and Agarwal, A. (2013) MQoSR: A Multiobjective QoS Routing Protocol for Wireless Sensor Networks. ISRN Sensor Networks, 2013, Article ID: 495803. http://dx.doi.org/10.1155/2013/495803

[25] Huang, X. and Fang, Y. (2008) Multiconstrained QoS Multipath Routing in Wireless Sensor Networks. Wireless Networks, 14, 465-478. http://dx.doi.org/10.1007/s11276-006-0731-9

[26] Radi, D. (2008) Favor of Improving Quality of Service in Wireless Sensor Thunderstruck Using Multiple Routing. Quarterly-Scientific Research Chamber of Electrical Engineering. 
Scientific Research Publishing (SCIRP) is one of the largest Open Access journal publishers. It is currently publishing more than 200 open access, online, peer-reviewed journals covering a wide range of academic disciplines. SCIRP serves the worldwide academic communities and contributes to the progress and application of science with its publication.

Other selected journals from SCIRP are listed as below. Submit your manuscript to us via either submit@scirp.org or Online Submission Portal.
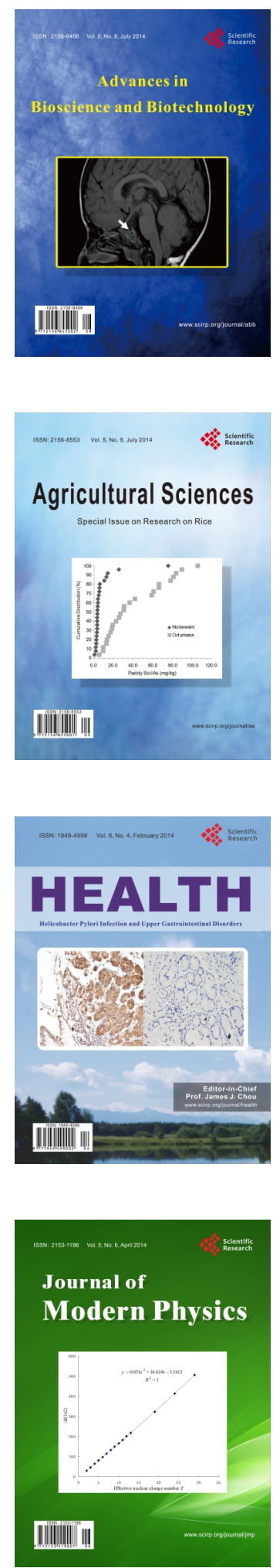
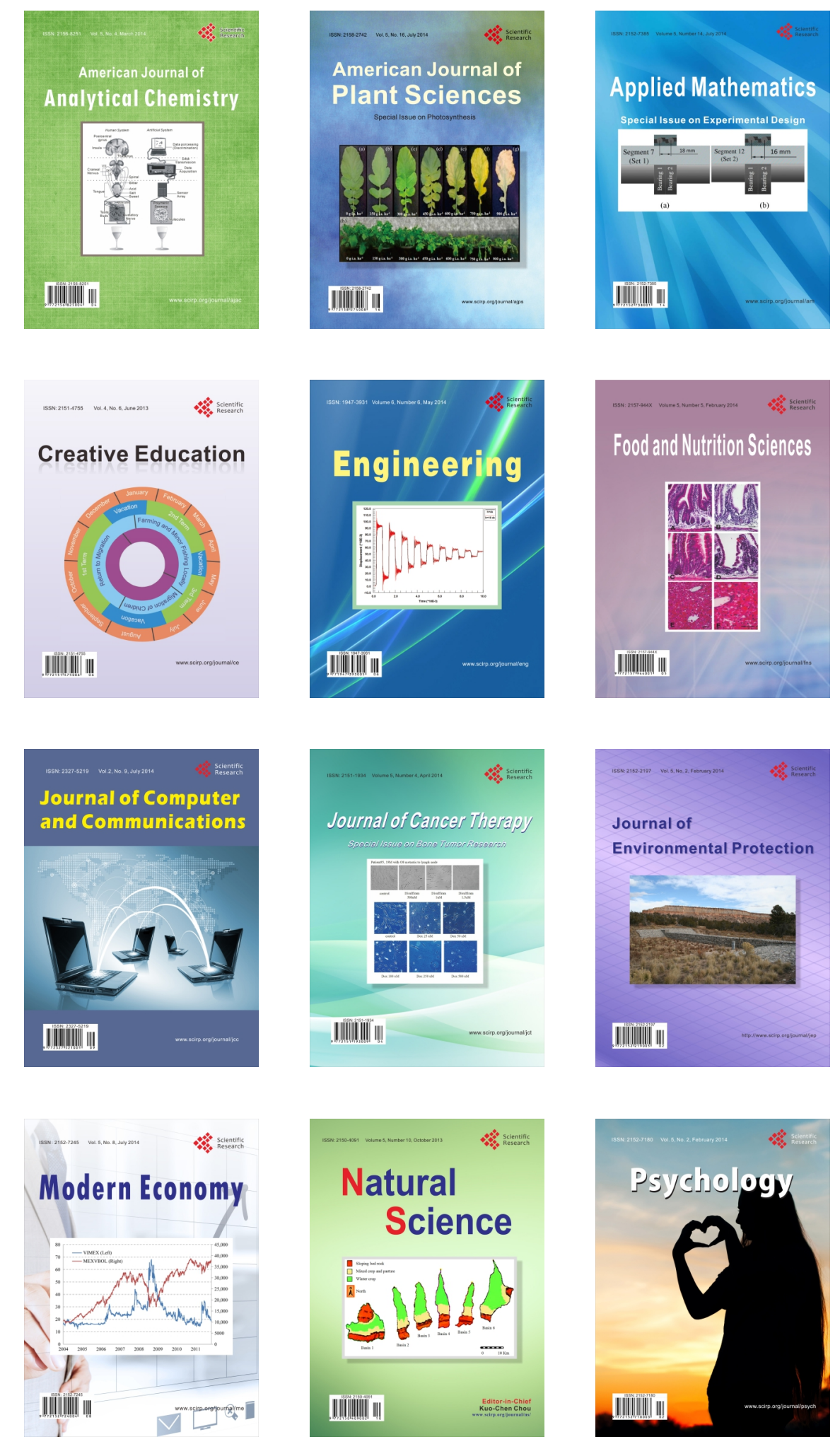